\title{
An Evacuation Route Model of Crowd Based on Emotion and Geodesic
}

\author{
Bangquan Liu $\mathbb{D},{ }^{1,2}$ Zhen Liu $\mathbb{D}^{2},{ }^{2}$ Dechao Sun $\mathbb{D}^{3},{ }^{3}$ and Chunyue Bi $\mathbb{D}^{3}$ \\ ${ }^{1}$ College of Information Engineering, Ningbo Dahongying University, Ningbo, China \\ ${ }^{2}$ Faculty of Electrical Engineering and Computer Science, Ningbo University, Ningbo, China \\ ${ }^{3}$ College of Electronics and Computer, Zhejiang Wanli University, Ningbo, China \\ Correspondence should be addressed to Zhen Liu; liuzhen@nbu.edu.cn and Dechao Sun; sundechao123@163.com
}

Received 27 July 2018; Accepted 12 September 2018; Published 1 October 2018

Guest Editor: Carlos Llopis-Albert

Copyright (c) 2018 Bangquan Liu et al. This is an open access article distributed under the Creative Commons Attribution License, which permits unrestricted use, distribution, and reproduction in any medium, provided the original work is properly cited.

Making unconventional emergent plan for dense crowd is one of the critical issues of evacuation simulations. In order to make the behavior of crowd more believable, we present a real-time evacuation route approach based on emotion and geodesic under the influence of individual emotion and multi-hazard circumstances. The proposed emotion model can reflect the dynamic process of individual in group on three factors: individual emotion, perilous field, and crowd emotion. Specifically, we first convert the evacuation scene to Delaunay triangulation representations. Then, we use the optimization-driven geodesic approach to calculate the best evacuation path with user-specified geometric constraints, such as crowd density, obstacle information, and perilous field. Finally, the Smooth Particle Hydrodynamics method is used for local avoidance of collisions with nearby agents in real-time simulation. Extensive experimental results show that our algorithm is efficient and well suited for real-time simulations of crowd evacuation.

\section{Introduction}

With the rapid development of society, densely populated places are increasing, and the security safety of the crowds is becoming more and more prominent, and the crowd trampling is an extreme event in natural crowd motion. Due to time and space constraints, it is difficult to complete the whole process of experimental exercises in the real life. Evacuation simulation is using computer to establish an effective simulation scene model to help solve the crowd evacuation behaviour in public environment. It is of great significance to prevent and deal with all kinds of major public safety incidents and reduce personal injury and economic loss [1]. In the real world, the emotional state of an individual plays a vital role in his/her decision-making and is easily influenced by others emotions. This process can be called emotional contagion [2]. In a specific group environment, emotional contagion will have an important influence on individual's emotions, behaviours, and decisions [3]. For example, during the emergency evacuation of a fire accident, emotional contagion will exacerbate the panic of the individual and prompt an individual to take immediate evacuation measures to reduce the time. Traditional multiagent navigation algorithms, like Reciprocal Velocity Obstacles (RVO) [4], have not considered the emotion of individuals. Emotional Reciprocal Velocity Obstacles (ERVO) model [5] improved the RVO with integrating the emotional effect into velocity decision, but it is limited for path planning. Path planning is one of the core technologies of evacuation simulation, which is used to simulate the behaviour of agents in the emergency scene. With the development of crowd simulation technology, although the traditional path planning method has been improved, the real-time path planning in evacuation simulation still faces many challenges.

Inspired by the above challenge, we proposed a novel evacuation simulation approach based on emotion and geodesic. Specifically, we first discussed the construction of individual emotional models in the process of emotional impact, where the individual emotional intensity, emotional infection, and emotional attenuation in evacuation process are described in detail. Based on the emotional impact method, we can add environmental information into a triangular mesh. By using the optimization-driven geodesic approach on triangle meshes, we could realize path planning 
in evacuation simulation. The evacuation path can be calculated in real time according to the dynamic state environment information. A complete evacuation simulation system is implemented, and the simulation experiments are carried out for different scenes. The experimental results verify the effective of the proposed model.

\section{Related Work}

In recent years, different types of evacuation simulation models have been proposed to simulate the behavior of evacuation of pedestrians in emergency situations [6-8]. According to the different objects of concern, these models can be divided into macroscopic $[9,10]$ and microscopic models [11-15]. The former is concerned with evacuating as a whole, assuming that all pedestrians have the same behavior pattern in the evacuation process. The latter is concerned with the simulation of individual behavior of pedestrians, which can generate different individual evacuation details. Compared with the macro model, the micro model can reproduce the influence of individual behavior on the evacuation process and make the simulation results truer. Therefore, the model gradually becomes the mainstream of the current evacuation simulation at home and abroad. This paper is in the micro frame. Under the frame, we focus on the path planning problem in evacuation simulation. Environmental modelling is the preparation of path planning. The evacuation environment is abstracted as a navigation map. The navigation map is usually represented as a graph structure composed of nodes and arcs. Different modelling methods $[16,17]$ can generate different navigation maps. Path planning means searching a path from the initial node to the target node on the map. In fact, the shortest path planning has become a widely used path planning method. There have been a lot of works on the shortest path planning in the fields of computer and robot. The Floyd algorithm, Dijkstra algorithm, and $\mathrm{A} *$ algorithm and its variant [18] can effectively find a shortest path on the navigation map.

However, when in a dangerous scene, pedestrians cannot find the shortest path under the influence of many factors and choose the safe area to move forward, or even ignore the optimal escape route to follow the flow passively [11]. The shortest path method is no longer applicable. In order to solve this problem, the extra decision module is introduced to evacuation simulation $[15,19,20]$. The decision module confirms the current subgoal according to the current status of the virtual human and then searches for a shortest path to the target. After arriving at the target, the decision module will continue to choose the next target and repeat until it reaches the security exit. This method can produce a good result when the rule is sufficient and reasonable. But the rule set used by the decision must fully reflect the characteristics of the crowd evacuation behavior under different circumstances and avoid the conflict among different rules. In addition, the local path planning among the subtargets is constantly carried out, ignoring the influence of the global environment information on the path selection.

Compared with the explicit formulation of rules set, the path of individual behavior can be naturally planned by introducing the influence of different factors in the scene directly into the path search process. Toll et al. [21] considered the dynamic population density of various regions in the field environment, and the path of virtual human planning can bypass the crowded area. Jaklin et al. [22] provided a method which can modify the initial path of the plan according to the role identity, and simulate the preference of different individuals to different terrain. The influence of individual emotion on its choice of evacuation path was also reflected in the path planning $[23,24]$. The above works considered individual differences in the path planning of pedestrians from different aspects. There is also lack of a path planning method which can take into account the individual psychology, the cognition of the scene, and the ability to act autonomously.

Compared with the real-time path planning method, the optimization method pays attention to the generation of the optimal path in evacuation. This kind of method establishes the optimization function with factors which need to be considered in evacuation and iteratively optimizes it to solve the optimal evacuation route. Usually, the evacuation time is the primary consideration. In recent years, the constraints of the path complexity are also taken into account $[1,25]$.

The above work can obtain the optimal path to satisfy various constraints; however, the path obtained by the optimization method will be restricted by the constraints considered. Even if sufficient constraints are added, the path generated by this method is too ideal, while the pedestrian in the reality is lacking in the panic and the global environment in the emergency scene. It is difficult to follow such a perfect path, so this kind of method is more suitable for the design of safety route. In addition, the optimization method needs to be solved iteratively. It takes a lot of time to calculate, it is difficult to achieve real time, and it is not suitable for real-time evacuation simulation.

\section{Materials and Methods}

3.1. Emotional Impact from Hazards and Agents. During the evacuation process, people often choose to stay away from dangerous sources so as not to cause injury to them. The environmental information of pedestrians will directly affect the planning of evacuation routes. We use triangulated mesh to create environmental information and specify a density function to represent the crowd density and information of hazard which will be updated dynamically during the simulation process. The dangerous value of sources to agents is limited, the closer they are to source, the danger is higher, and, to a certain region, the threat decreases rapidly. Assume that the dangerous area is circular, the Gauss function can be used to calculate the hazard value $\psi_{i}$ of $p_{i}$ :

$$
\psi_{i}=\frac{1}{\sqrt{2 \pi}} \exp ^{-\left(v_{i}-v_{0}\right)^{2} / 2} \times \psi_{0}
$$

where $v_{i}$ is the $i$-th vertex of the mesh, $v_{0}$ is the source vertex of hazards on the mesh, and $\psi_{0}$ is the danger value in hazard source $v_{0}$.

In this paper we use triangulation grid to establish navigation grid and record the area $s$ of each triangle. According to the position of agents, we can compute the number of agent 
in each triangle, then the crowd density of triangle can be calculated using the following:

$$
\rho=\frac{\sum \pi r_{i}^{2}}{s}
$$

where $r_{i}$ is the radius of agent which is in the crowd. $s$ is the area of the triangle in which the crowd is located. After computing the density, the crowd density information of surface will be stored in the triangle mesh.

Agents are easily affected by the environment and dangerous information in the process of movement; the closer they are to the hazard, the stronger the agent's willingness to escape the hazard is. At the same time, agents have different reactions to the crowd based on their emotions and environmental factors. In a group environment, the individual is bound to be affected by emotion, but the degree of influence is different. The reason is that the individual has different emotional preferences, the ability to capture emotional clues, and emotional feeling. The group with the same purpose is in the same situation. Although the individual has different degrees of cognition and evaluation to the same stimulus, the emotion of the experience is consistent, only the degree is different. In the calculation of emotional impact, we mainly consider two sources of emotion: special individual emotion and the emotions created by the group. The emotional contagion value $I_{i}(t+1)$ of the $i$-th agent $A G_{i}$ at the time of $T+1$ is

$$
\begin{aligned}
I_{i}(t+1)= & P_{i}^{t} \times\left(\omega \times \psi_{d}+(1-\omega) \times G E \times I_{i}(t)\right) \\
& +I_{i}(t)
\end{aligned}
$$

where $I_{i}(t)$ is the emotional value of the $A G_{i}$ at the time of $T$ and $\omega$ is an individual's attention to the danger value $\psi_{d}$ of hazard source in the current position. $P_{i}^{t}$ is the optimistic mood at the time of $t$, expressing the ability of individual emotion to feel positive influence from other groups. $G E$ is the group emotion, defined as follows:

$$
G E=\sum\left(\beta_{i} \times \mu_{i} \times I_{i}(t)\right)
$$

Although crowd emotion is composed of individual emotions, it is not simply a cumulative accumulation of individual emotions. $\beta_{i}$ is the leadership coefficient which indicates the influence of $A G_{i}$ 's emotion on group emotion. $\mu_{i}$ defines the influence of Agent $_{i}$ on group emotion in a group, and the formula is as follows:

$$
\mu_{i}=\frac{P_{i}^{t}}{\sum_{i \in G} P_{i}^{t}}
$$

Personality is the unique mental and psychological characteristics of individuals formed in social activities and is different from other individuals. The possible emotional states of $A G_{i}$ include negative emotional state and positive emotional state. Agents who are in positive emotional state are calmer and easier to integrate into groups to reach the destination quickly. The emotion of the agent will be changed with the emotion of the surrounding, in easy step of simulation process, the optimistic emotion $P_{i}^{t}$ of $A G_{i}$ also will be updated with a certain randomness, and the formula is as follows:

$$
P_{i}^{t+1}=P_{i}^{t} \times\left(1+\alpha_{p}\right)
$$

where $\alpha_{p}$ is the change value of indicates optimistic emotion, which can be calculated by the following formula:

$$
\alpha_{p}=\frac{\sum_{i=1}^{N_{p}} \lambda_{p}(i)}{N_{p}} \chi_{p}
$$

where $N_{p}$ is the number of agents who could affect the emotion of $A G_{i} \cdot \chi_{p}$ represents the coefficient factor of an agent's optimism. $\lambda_{p}(i)$ indicates the optimistic emotion value from other agents around $A G_{i}$, and it can be calculated by the following formula:

$$
\lambda_{p}(i)=(1-\eta) \times(1-\rho)
$$

where $\rho$ is the crowd density of triangle that can be calculated by (2). The weight value $\psi$ of an agent that has emotions is calculated using the following:

$$
\eta=\frac{\alpha}{1+B e^{b \omega}}
$$

$\eta$ takes a value in the range $[0,1] . \omega$ is composed of two emotions. The two emotions, which are orthogonal dimensions of the emotion space and selected from the OCC model [26], are hope and fear. An agent's emotion is a twodimensional vector, where each dimension is represented by an emotion:

$$
\Pi=\left\langle\psi_{H}, \psi_{F}\right\rangle
$$

The variable $\omega$ of (9) can be calculated by the following:

$$
\omega=\psi_{H}-\psi_{F}
$$

where $\psi_{H}+\psi_{F}=1, \psi_{H}$ is the hope emotion and $\psi_{F}$ is the fear emotion. Generally, agents will choice a suitable way to escape the dangerous area based on their own emotional state and environmental information. There are always two ways: one is to join an appropriate group and follow the leader to evacuate, and the other is to choose the shortest path to move away from the dangerous area and avoid the crowd. When the agent meets a crowd, the value of the agent following the crowd is calculated as follows:

$$
T_{\text {flow }}=\frac{Q}{\sum_{i=1}^{N_{p}} I_{i}(t)}
$$

$Q$ is an adjustment coefficient. The way to update the action of an agent can take the following rules: if $T_{\text {flow }}<P^{t}$, the agent will keep away from the crowd and take the crowd as an obstacle to recalculate the appropriate path. If $T_{\text {flow }}>P^{t}$, the agent will back out the original path planning and join into the crowd and evacuate with others.

3.2. Geodesic Path with Non-Uniform Density. Let $\mathcal{S}$ be a triangulated polyhedral surface in $\mathscr{R}^{3}$, defined by a set of vertices, edges, and faces. Given two points $s, t \in \mathcal{S}$, there are infinitely many paths between them. Our task is to calculate the shortest path that passes the obstacles and meet other 


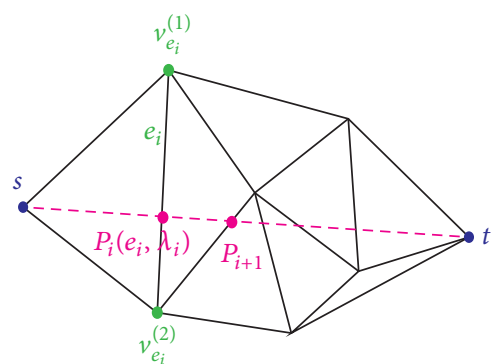

Figure 1: A discrete path $\left(s, p_{i}, p_{i}+1, \ldots, p_{k}, t\right)$ can be encoded using the face sequence.

given requirements. Let us assume the mesh with obstacles have different density with the other base mesh. We use the optimization-driven approach [27] to compute geodesic paths on triangle meshes with nonuniform density setting. Let us assume $v_{e_{i}}^{(1)}$ and $v_{e_{i}}^{(1)}$ are the endpoints of the edge $e_{i}$, as shown in Figure 1; the initial path (shown in red) through the face sequence $\Gamma$ can be described by $\left(s, p_{1}, p_{2}, \ldots, p_{k}, t\right)$, where $p_{i}$ is the intersection point between the geodesic path and the $i$-th edge $e_{i} \triangleq\left(v_{e_{i}}^{(1)}, v_{e_{i}}^{(2)}\right)$ in the face sequence. Then $p_{i}$ can be represented by a scalar $\lambda_{i} \in[0,1]$ so that $p_{i}=$ $\left(1-\lambda_{i}\right) v_{e_{i}}^{(1)}+\lambda_{i} v_{e_{i}}^{(2)}$.

We will discuss the case where the base mesh has a nonuniform density function defined on the surface, as Figure 2 shows. Typically, each polygonal face $f_{i}$ has a constant density value $\rho\left(f_{i}\right)$. In this case, the total path length is given by

$$
\begin{aligned}
L\left(\Gamma ; \lambda_{1}, \lambda_{2}, \ldots, \lambda_{k}\right)= & \rho\left(\overrightarrow{s p_{1}}\right)\left\|\overrightarrow{s p_{1}}\right\| \\
& +\sum_{i=1}^{k-1} \rho\left(\overrightarrow{p_{i} p_{i+1}}\right)\left\|\overrightarrow{p_{i} p_{i+1}}\right\| \\
& +\rho\left(\overrightarrow{p_{k} t}\right)\left\|\overrightarrow{p_{k} t}\right\|,
\end{aligned}
$$

whose gradient with regard to $\lambda_{i}$ is

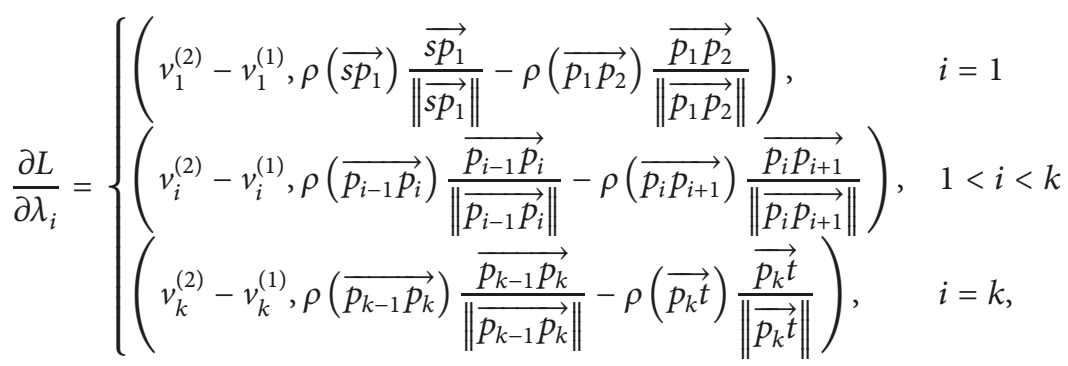

where $\rho(\overrightarrow{A B})$ denotes the density in the face containing the line segment $\overrightarrow{A B}$. It is easy to know that the path tends to go through the area with a low density. The density value $\rho\left(f_{i}\right)$ of the affected triangle meshes around the hazard source $p_{0}$ can be calculated by (1). If there is a crowd in scene, the density $\rho$ of triangle $f_{i}$ can be computed using (2). When the agent meets the hazards or crowds, it will update moving path according to its self-emotion, crowd emotion, and the new geodesic path. The pseudocode of computing geodesic is shown in Algorithm 1.

3.3. Collision Avoidance. Collision avoidance is a way to avoid collisions with other nearby individuals by adjusting their own moving velocity. In the evacuation simulation, these interactions including attraction, repulsion, and friction play key roles in agent evacuation behaviours, speed, and efficiency. It is necessary to adjust the local position of each agent by combining the potential force toward the target and the interaction force among agents to achieve collision avoidance. The final force of agent to control the moving can be calculated as follows:

$$
\begin{aligned}
F\left(X_{i}\right)= & \frac{1}{\left\|p\left(X_{t}\right)-p\left(X_{i}\right)\right\|} \times F_{\text {dirve }}\left(X_{i}\right) \\
& +\left\|p\left(X_{t}\right)-p\left(X_{i}\right)\right\| \times F_{\text {repulse }}\left(X_{i}\right)
\end{aligned}
$$

where $p\left(X_{i}\right)$ is the local position of agent $_{i}$ and $p\left(X_{t}\right)$ is the target position, and $F_{\text {dirve }}\left(X_{i}\right)$ is the potential force of agent ${ }_{i}$ moving to the target position. $F_{\text {repulse }}\left(X_{i}\right)$ is the interaction force between each agent is calculated by using the Smoothed-particle hydrodynamics (SPH) method proposed by Gingold and Monaghan [28, 29]. SPH is a well-known computational method used for simulating the mechanics of continuum media, such as solid mechanics and fluid flows. Unlike grid-based techniques, SPH creates a free surface and pressure from weighted contributions of neighbouring particles rather than by solving linear systems of equations. The reciprocal influence between each agent is equal to the weighted average value of the interaction forces among agents in their scope of view. The interaction force $F_{\text {repulse }}\left(x_{i}\right)$ of agent $_{i}$ on position $x_{i}$ can be calculated by Coulomb's law [30].

$$
F_{\text {repulse }}\left(X_{i}\right)=\sum_{k=1}^{K} m_{k} \frac{F_{\text {repulse }}\left(x_{k}\right)}{\rho_{k}} W\left(x_{i}-x_{k}\right)
$$

where $W$ is a weight function, also could be called smooth kernel function and be expressed as follows:

$$
W\left(x_{i}-x_{k}\right)=\frac{\alpha e^{-\left\|p\left(x_{i}\right)-p\left(x_{k}\right)\right\| / \beta}}{\left\|p\left(x_{i}\right)-p\left(x_{k}\right)\right\|}
$$

$\alpha$ and $\beta$ are constant coefficients and $p\left(x_{k}\right)$ is the position of other agent $_{k}$ who can interact with agent $t_{i}$ in feasible 


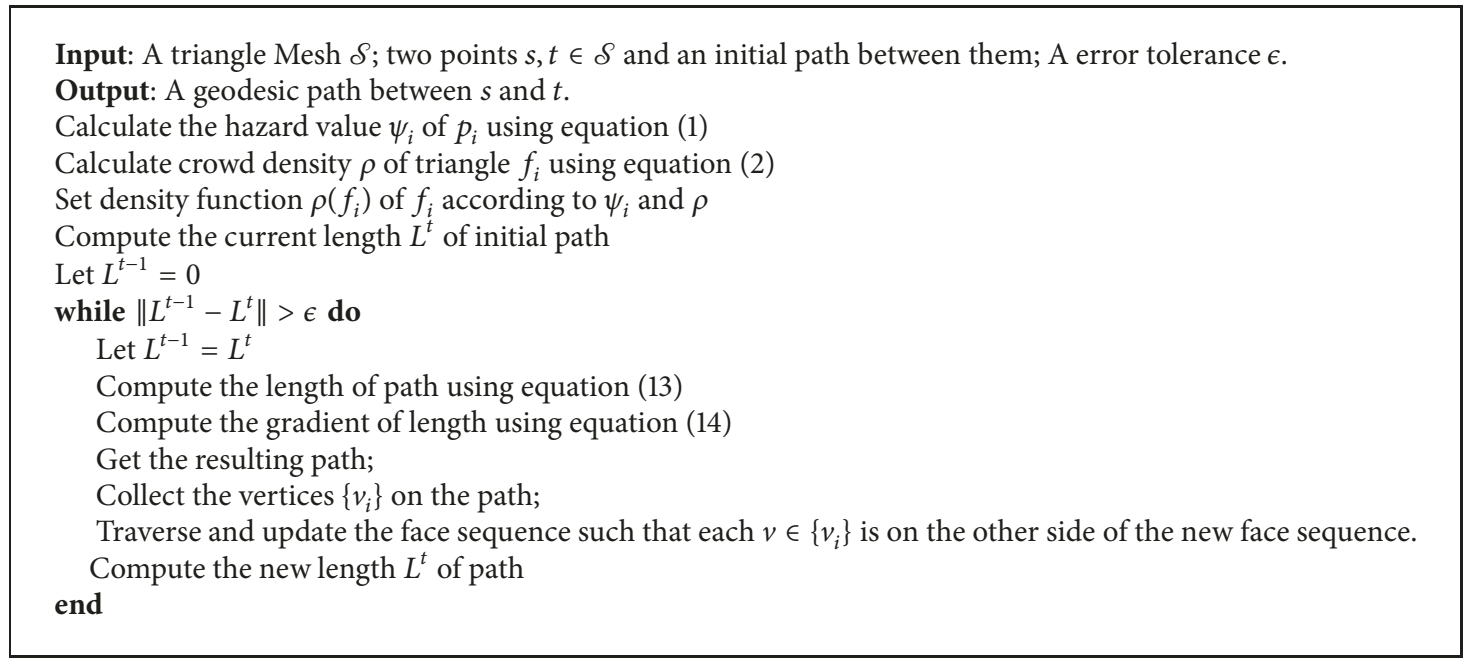

Algorithm 1: An optimization based method for computing the shortest path with hazard information.

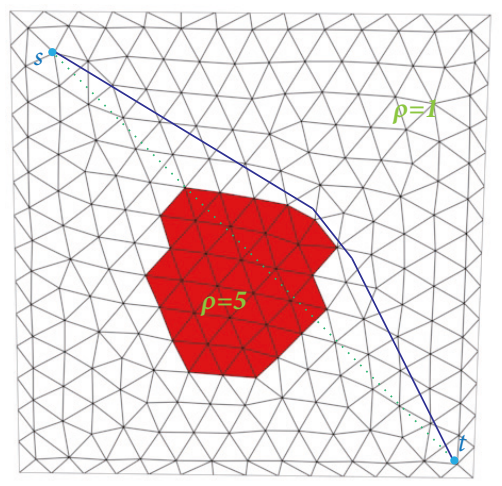

FIGURE 2: The green path is geodesic path assuming the density is uniform on the surface, while the blue path is geodesic path in the density $\rho=5$ set on red faces and the other with $\rho=1$.

range. In our experiment of crowd evacuation simulation, taking $\alpha=1, \beta=1$. When using the $\mathrm{SPH}$ method to calculate the force, it is important to search other agents in the agent's scope and the time complexity is $O\left(n^{2}\right)$, where $n$ is the number of agents. The computational efficiency decreases slowly with the increasing rate of $n$. In order to improve the computational efficiency, we use the Approximate Nearest Neighbor (ANN) method [31] to find the agents in its selfscope.

\section{Results and Discussion}

We implemented and experimented with our algorithm on a computer with a 64-bit version of Win7 system, a $3.07 \mathrm{GHz}$ Intel(R) Core(TM) i7 CPU, and 6 GB memory. The coding language is $\mathrm{C}++$. We shall use extensive experimental results to exhibit demonstrate the algorithm's efficiency, as well as its insensitiveness to scene resolution, and its robustness to small surface variations and topology. To demonstrate
TABLE 1: Timing statistics (seconds) for geodesic computation on a workstation with a $3.07 \mathrm{GHz}$ CPU and 6GB RAM.

\begin{tabular}{lccc}
\hline scenario & \#Faces & Average time(s) & Max time(s) \\
\hline scene1(Figure 3) & 2,000 & 0.05 & 0.08 \\
scene1(Figure 3) & 10,196 & 0.11 & 0.19 \\
scene1(Figure 3) & 51,265 & 0.34 & 0.40 \\
scene1(Figure 3) & 105,767 & 0.64 & 0.41 \\
scene2(Figure 4) & 20,852 & 0.15 & 0.20 \\
scene3(Figure 5) & 12,987 & 0.11 & 0.15 \\
scene4(Figure 6) & 25,632 & 0.17 & 0.19 \\
scene5(Figure 7) & 22,572 & 0.15 & 0.21 \\
\hline
\end{tabular}

specific features and analyze capability of our method, we carry out the evacuation simulation in different scenarios.

To demonstrate the path planning algorithms efficiency, we developed a program to automatically generate triangulated mesh for scenes and simple interface for users to interactively specify obstacle regions. The radius $\gamma_{i}$ of agent is set to 3; other parameters in our system are set experimentally: $\omega=0.5, \varphi_{0}=20, Q=2, B=1, \alpha=$ $2, b=5$. To measure the geodesic algorithm performance, we randomly sample $1 \mathrm{~K}$ pairs of vertices to take down the average computation time and the max computation time. As shown in Table 1, for scene with around $20 \mathrm{~K}$ faces, our algorithm takes 0.15 s averagely to compute a geodesic path and can be used for real-time path planning.

4.1. Evacuate Simulation under Different Scenarios. Our method can handle mesh information changes during the simulation. Taking a simple scenario shown in Figure 3 as an example, a group evacuate from sources to targets with the geodesic path which is calculated based on obstacles and hazard information. This scene has $300 \times 300$ units with $5 k$ triangulated faces and two exports. The group with a leader move from the source position $s$ to the target position $t$, respectively. As shown in Figure 3(a), there are some obstacles 


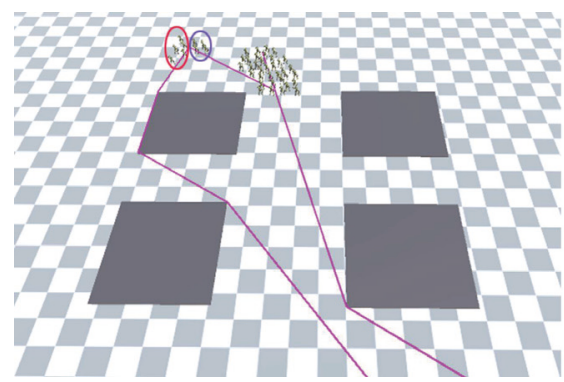

geodesic paths

1st frame

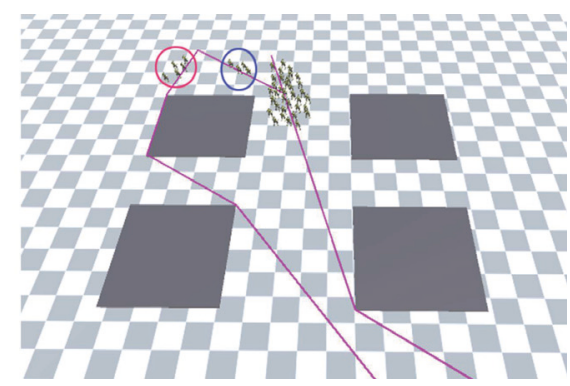

12th frame

15th frame

(a) Without hazards

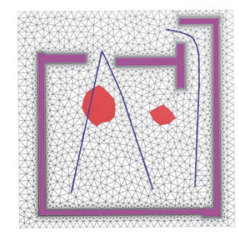

geodesic paths

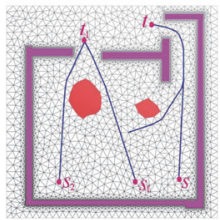

geodesic paths

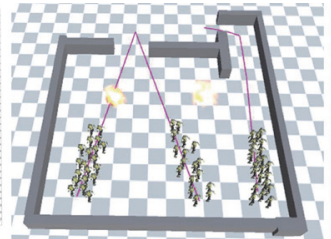

1st frame

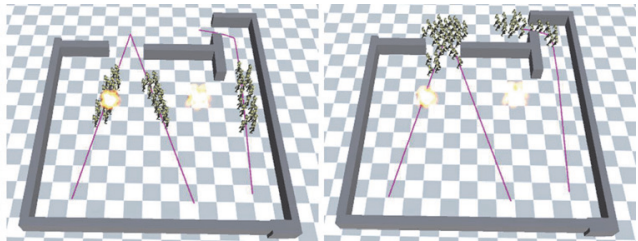

12th frame

15th frame (b) With hazards and without emotional impact

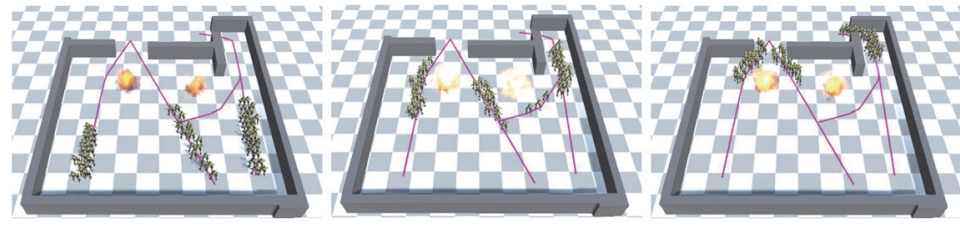

12th frame

15th frame

(c) With hazards and emotional impact

FIGURE 3: Snapshots of crowd simulation in the same scenarios with different hazard and emotional impact. Images in the left line represent the geodesic paths on the triangle mesh. The other three images represent the different snapshots of the simulation process at 1st, 12th, and 15 th frame, respectively.

in the scene; we set density $\rho=5$ in the region where the obstacles are located and can get the geodesic path in realtime. The geodesic is the shortest path, so the crowd can reach their destination as quickly as possible under their own speed. In Figure 3(b), we add an explosion hazard in the region and agents without emotion will keep moving along the original planned directions. In Figure 3(c), we add the sample hazard into scene and agents with emotion impact, the agents will recalculate their moving path to escape away from the hazard source. We also specify a dangerous factor $\rho=10$ to the affected triangulation faces and can get the safety geodesic path less than 0.1 seconds. The simulation results show that the crowd completely avoids the hazards and obstacles.

We also simulate evacuation behaviors in a crowd with the following hazard situations: persistent hazards occur at different moments time. The simulation runs in a simple maze field with two exits. Figure 4 shows some snapshots of crowd movement in this simulation. All the agents move randomly at the beginning of simulation. If a persistent hazard occurs at a specific time as shown in the 10-th frame of Figure 4(a), those agents will keep far away from the hazards continually as long as they evacuated to an exit along the geodesic path. When another persistent hazard occurs at the evacuating exit as shown in the 20-th fame of Figures 4(a) and 4(b), all the agents will escape away from both of the hazards and choose to evacuate from another safe exit.

In order to validate the effectiveness of individual emotions and group information, we run crowd simulations in a scene with a crowd and agents with different emotions. There are four obstacles in the scene and each agent has different emotional values $\varphi_{H}$ and $\varphi_{F}$. The agents with $\varphi_{F}=0.6$ and $\varphi_{H}=0.4$ in red circle choose to stay away from the crowd for evacuating. We use (2) to set the crowd density value $\rho$ in the region where the crowd is located when calculating the geodesic path for those agents. In Figure 5, when the agents in the red circle see a group of crowd in scene, the crowd is seen as an obstacle, and the geodesic path is recalculated for evacuating. On the contrary, the agents with $\varphi_{F}=0.3$ and $\varphi_{H}=0.7$ in the blue circle choose to join the crowd and move forward with the crowd.

4.2. Analysis of Emotional Impact. Some simulations of crowd movement with and without emotion model, shown in Figure 6, are used to validate the effectiveness of emotional impact in our method. As shown in Figure 6(a), agents without emotion will keep moving along the geodesic path rendered in red line. In contrast, the agents with emotion will replan the evacuation geodesic path to escape away from the 


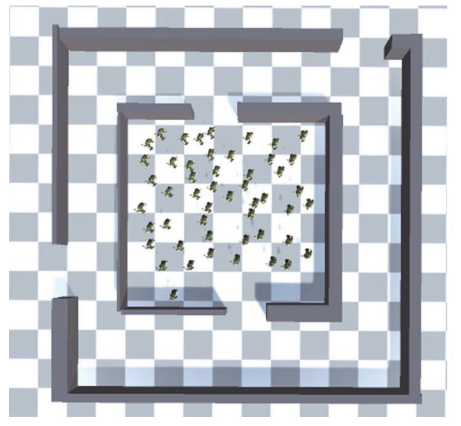

1st frame

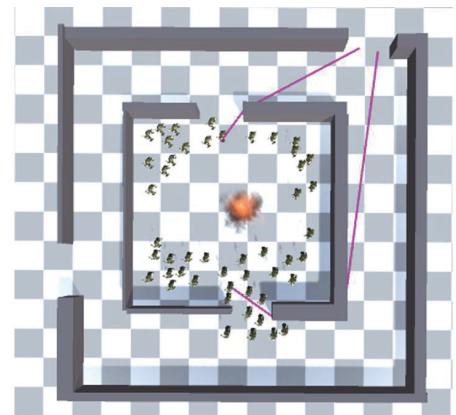

10th frame

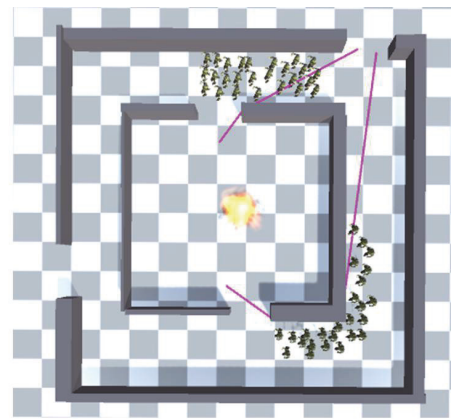

20th frame

(a) A persistent hazard occurs in a position

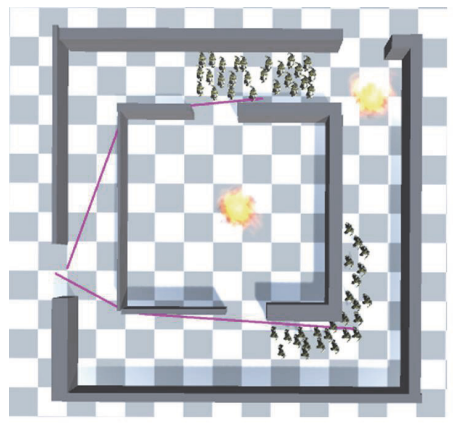

20th frame

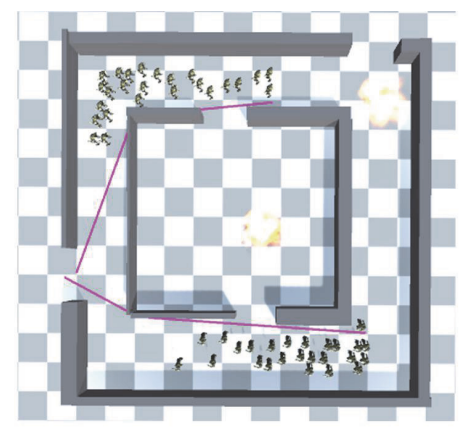

35th frame

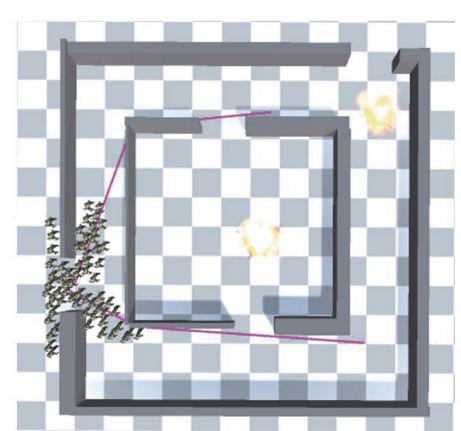

50th frame

(b) Another persistent hazard added into the scene

FIGURE 4: Snapshots of crowd simulation in different types of hazard scenarios.

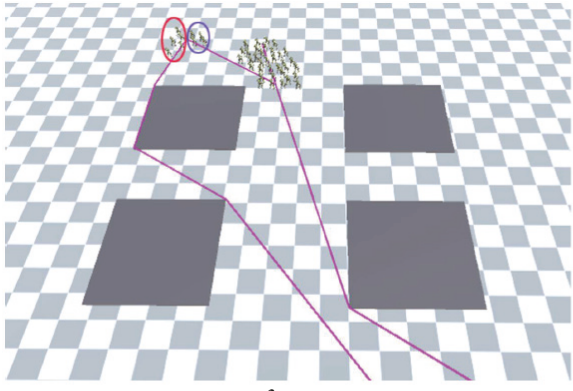

1st frame

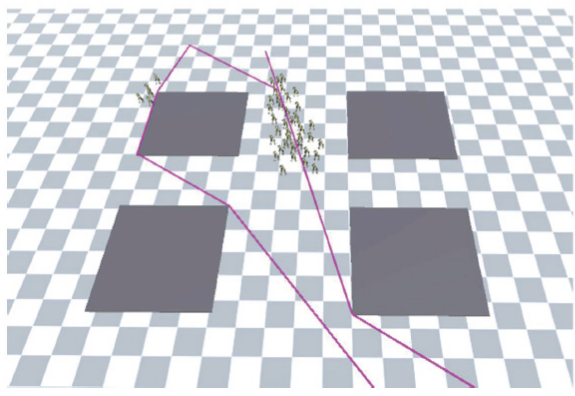

18th frame

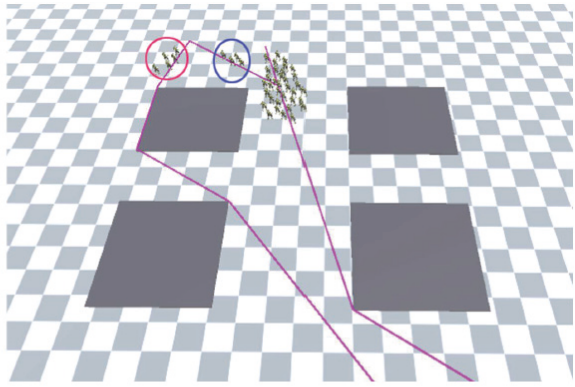

8th frame

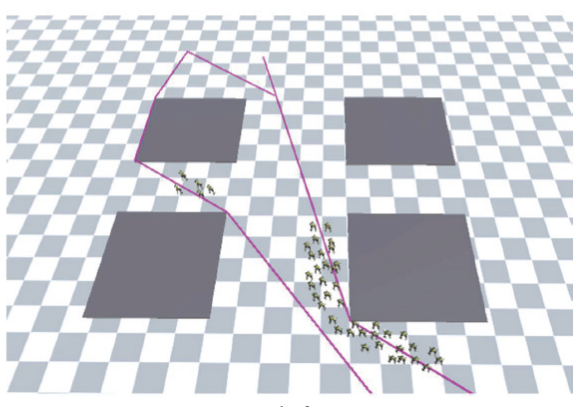

25th frame

FIGURE 5: Agents with different hope emotion $\psi_{H}$ and fear emotion $\psi_{N}$ choose different evacuation when they meet another group. Four images represent the different snapshots of the simulation process at different moments. 


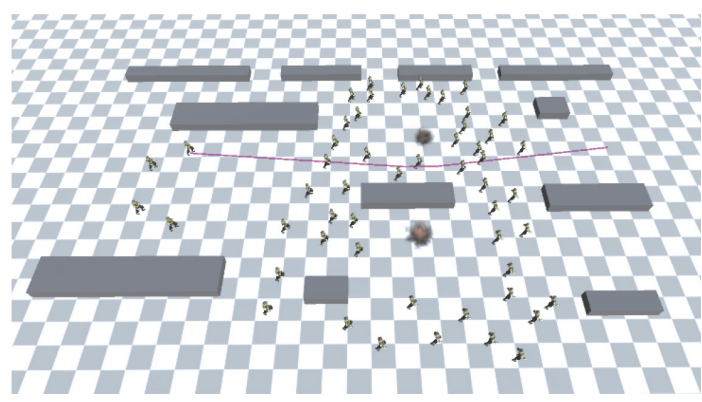

(a) Without emotion

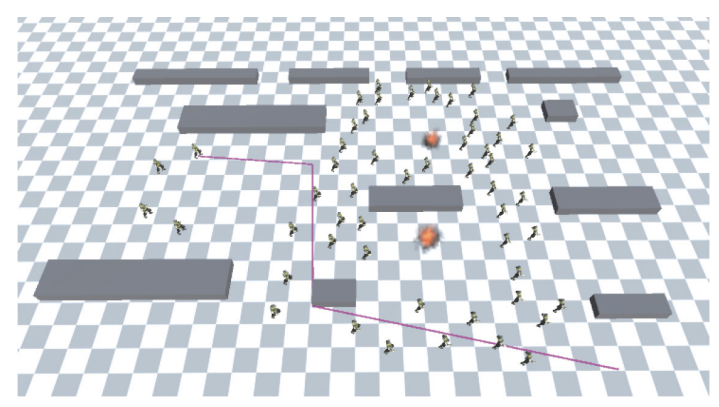

(b) With emotion

FiguRE 6: The comparison of crowd movements with and without emotion.

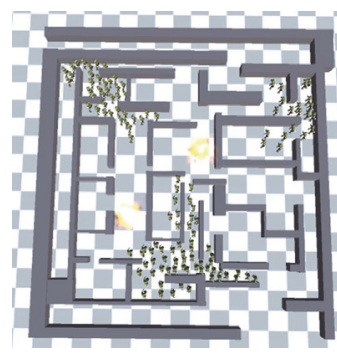

1st frame

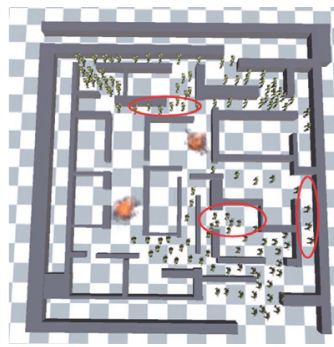

15 st frame

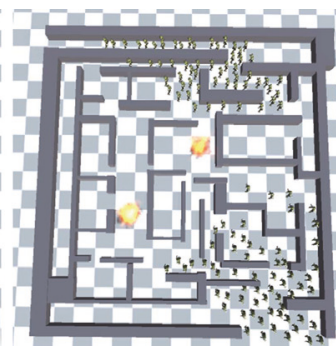

25 th frame

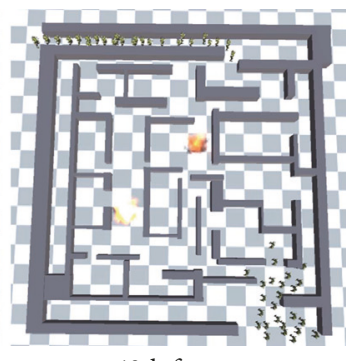

40th frame

(a) Evacuation simulation result by our approach in a maze

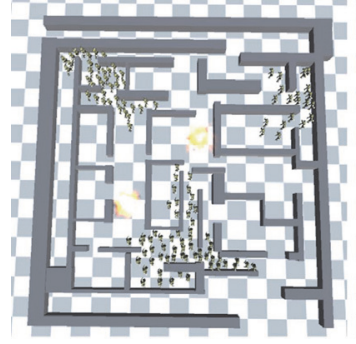

1st frame

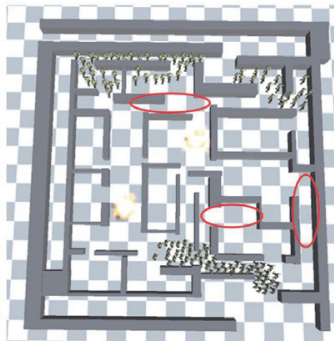

15 st frame

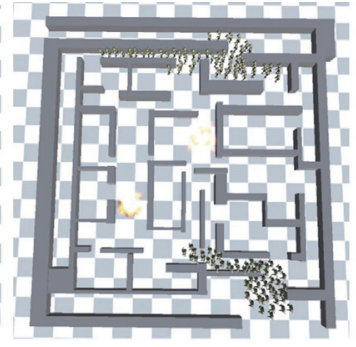

25th frame

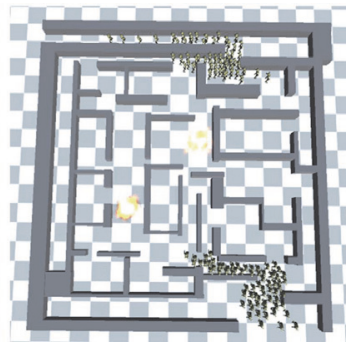

40th frame

(b) Evacuation simulation result by RVO [4] in a maze

FIGURE 7: Performance comparison. Our algorithm has a performance advantage compared with the existing algorithms.

hazards even when they have not reached the nearby region of the hazard, as shown in Figure 6(b). As we can see from Figure 6, the crowd movement in a hazard environment is affected by the panic emotion significantly.

4.3. Comparisons with Other Methods. We compare our algorithm with RVO algorithm in a maze scene with some hazards and multigroups. The effect in Figure 7(b) evacuation result using RVO method, Figure 7(a) is the result of using our algorithm. Figures $7(\mathrm{a})$ and $7(\mathrm{~b})$ are frames of the same time in the simulation process, they can be observed that if the RVO method is used, agents with similar positions tend to concentrate on the exit and cause blockage. By using our method, we can get all the possible paths in the scene by setting the emotional value of agents. The 40th frame of Figures $7(\mathrm{a})$ and $7(\mathrm{~b})$ shows that when the evacuation time comes to the end of the simulation, there is still a local blockage in the simulation results of RVO methods and the simulation with our algorithm is close to the end of evacuation.

We use the same scenario with different numbers of agents from 100 to 2000 to evaluate its performance and compared it with two representative methods: Reciprocal Velocity Obstacles (RVO) algorithm [4] and Emotion Reciprocal Velocity Obstacles (ERVO) algorithm [5]. Figure 8(a) shows the timing statistics and Figure 8(b) shows the Frames Per Second (FPS) statistics, from which we can clearly see a performance advantage compared with the existing algorithms, whether it is simulation time or FPS.

\section{Conclusions}

In this paper, we analyzed the importance of exploring the model of emotional impact and evacuation simulation. In 


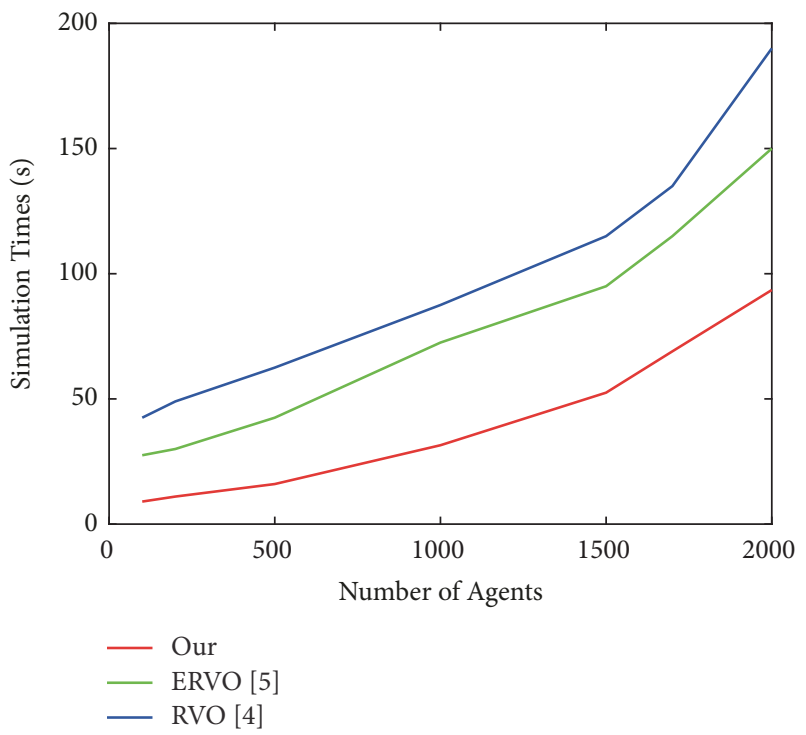

(a) Comparison of times

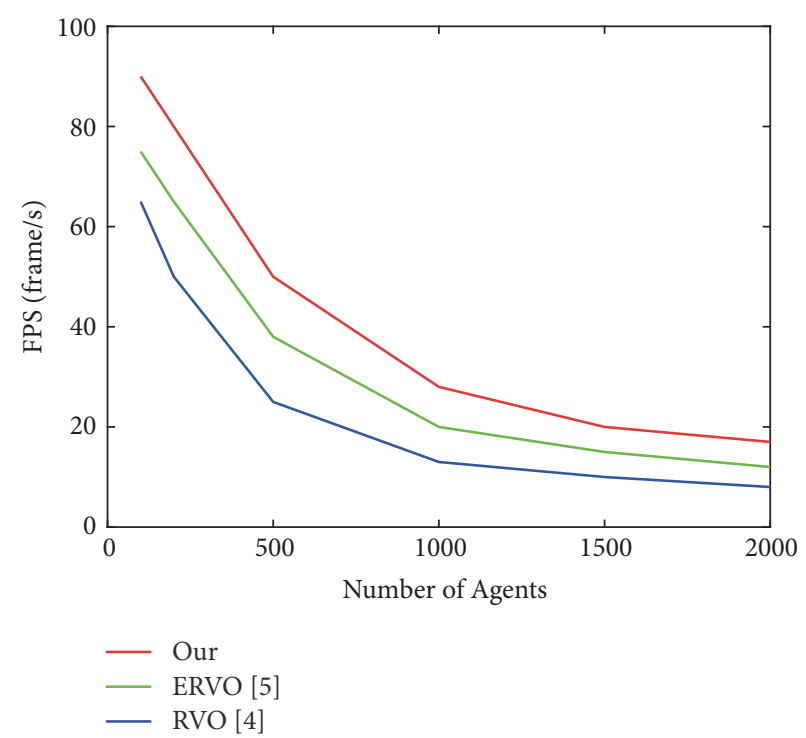

(b) Comparison of FPS

FIgURE 8: Performance comparison.

view of the deficiencies in the emotional simulation of the population, a model of crowd emotional evacuation is proposed from the perspective of social computing, and a realtime path planning method is proposed for the application of evacuation simulation. The results are as follows.

(1) In the process of constructing emotion model, we fully take into account the effect of personality, emotion, and crowd emotion. The influence of group emotion and special individual emotion on the emotional experience of virtual individuals is taken into account in the intensity of emotional infection.

(2) The individual emotion in the crowd is divided into two types of positive and negative emotions. The key to emergency management of the crowd needs to control the infection of negative emotions in the crowd and avoid the crowd in the negative state.

(3) Based on the emotion method, we can add environmental information into a triangular mesh and use the optimization-driven geodesic approach on triangle meshes, we can realize path planning in evacuation simulation.

The evacuation simulation of dense crowd is a challenging research topic, and there are many problems worthy of further study. The work of this paper is still to be further deepened. The next step is to combine computer vision technology to collect crowd motion parameters and supplement the experiment of crowd calculation.

\section{Data Availability}

The simulation data used to support the findings of this study are available from the corresponding author upon request.

\section{Conflicts of Interest}

The authors declare that they have no conflicts of interest.

\section{Acknowledgments}

This work is supported in part by the Science and Technology Plan Project of Zhejiang (LGG18F020001, 2017C33079), NSF of Zhejiang (LY17F020002), Philosophy and Social Sciences Project of Zhejiang (19NDJC0103YB), and NSF of Ningbo (2016A610040, 2016A610042, 2017A610120).

\section{References}

[1] Z. Ren, W. Gai, and Q. Peng, "Ego-centered path planning in evacuation simulation," Journal of Computer-Aided Design \& Computer Graphics, 2015.

[2] E. Hatfield, J. T. Cacioppo, and R. L. Rapson, "Emotional Contagion," Current Directions in Psychological Science, vol. 2, no. 3, pp. 96-100, 1993.

[3] Z. Liu, W. Jin, P. Huang, and Y. Chai, "An emotion contagion simulation model for crowd events," Computer Research and Development, vol. 50, no. 12, pp. 2578-2589, 2013.

[4] J. D. Van Berg, M. Lin, and D. Manocha, "Reciprocal velocity obstacles for real-time multi-agent navigation," in Proceedings of the 2008 IEEE International Conference on Robotics and Automation, ICRA 2008, pp. 1928-1935, Pasadena, Calif, USA, May 2008.

[5] M. Xu, X. Xie, P. Lv et al., "Crowd behavior simulation with emotional contagion in unexpected multi-hazard situations," Multiagent Systems, 2018.

[6] E. Ronchi, P. A. Reneke, and R. D. Peacock, "A conceptual fatigue-motivation model to represent pedestrian movement during stair evacuation," Applied Mathematical Modelling, vol. 40, no. 7-8, pp. 4380-4396, 2016

[7] X. Zheng, T. Zhong, and M. Liu, "Modeling crowd evacuation of a building based on seven methodological approaches," Building and Environment, vol. 44, no. 3, pp. 437-445, 2009.

[8] Z. Liu and P. Huang, "Study of panic behavior model for crowd on pedestrian bridge in emergent event," Journal of System Simulation, vol. 24, no. 9, pp. 1950-1953, 2012. 
[9] R. L. Hughes, "A continuum theory for the flow of pedestrians," Transportation Research Part B: Methodological, vol. 36, no. 6, pp. 507-535, 2002.

[10] R. M. Colombo and M. D. Rosini, "Pedestrian flows and nonclassical shocks," Mathematical Methods in the Applied Sciences, vol. 28, no. 13, pp. 1553-1567, 2005.

[11] D. Helbing, I. Farkas, and T. Vicsek, "Simulating dynamical features of escape panic," Nature, vol. 407, no. 6803, pp. 487490, 2000.

[12] A. Braun, B. E. J. Bodmann, and S. R. Musse, "Simulating virtual crowds in emergency situations," in Proceedings of the VRST'05 - ACM Symposium on Virtual Reality Software and Technology 2005, pp. 244-252, Monterey, Calif, USA, November 2005.

[13] C. Wang and Z. Hangzhou, "Physically based realistic simulation of crowd evacuation during a fire," Journal of ComputerAided Design Computer Graphics, vol. 20, no. 8, pp. 1033-1037, 2008.

[14] D. Sun, S. Xin, Y. Zhou, S. Chen, R. Wang, and Z. Shu, "Importance driven medial axis," Journal of Computer-Aided Design \& Computer Graphics, 2016.

[15] N. Pelechano and N. I. Badler, "Modeling crowd and trained leader behavior during building evacuation," IEEE Computer Graphics and Applications, vol. 26, no. 6, pp. 80-86, 2006.

[16] O. B. Bayazit, J.-M. Lien, and N. M. Amato, "Roadmap-based flocking for complex environments," in Proceedings of the 10th Pacific Conference on Computer Graphics and Applications, PG 2002, pp. 104-113, Beijing, China, October 2002.

[17] W. Van Toll, A. F. Cook, and R. Geraerts, "Navigation meshes for realistic multi-layered environments," in Proceedings of the 2011 IEEE/RSJ International Conference on Intelligent Robots and Systems: Celebrating 50 Years of Robotics, IROS'11, pp. 3526-3532, San Francisco, Calif, USA, September 2011.

[18] T. H. Cormen, C. E. Leiserson, R. Rivest, and C. Stein, "Introduction to algorithms (third edition)," Computer Education, vol. 39, no. 4, pp. 643-674, 2013.

[19] J. Joo, N. Kim, R. A. Wysk et al., "Agent-based simulation of affordance-based human behaviors in emergency evacuation," Simulation Modelling Practice and Theory, vol. 32, no. 2, pp. 99115, 2013.

[20] S. Zeng, Z. Mu, and T. Balezentis, "A novel aggregation method for pythagorean fuzzy multiple attribute group decision making," International Journal of Intelligent Systems, vol. 33, no. 3, pp. 573-585, 2017.

[21] W. G. Van Toll, A. F. Cook IV, and R. Geraerts, "Real-time density-based crowd simulation," Computer Animation and Virtual Worlds, vol. 23, no. 1, pp. 59-69, 2012.

[22] N. Jaklin, A. Cook IV, and R. Geraerts, "Real-time path planning in heterogeneous environments," Computer Animation and Virtual Worlds, vol. 24, no. 3-4, pp. 285-295, 2013.

[23] T. Donaldson, A. Park, and I. Lin, "Emotional Pathfinding," in Advances in Artificial Intelligence, vol. 3060 of Lecture Notes in Computer Science, pp. 31-43, Springer, Berlin, Germany, May 2004.

[24] A. Johansson and P. Dell'Acqua, "Pathfinding with emotion maps," in Intelligent Computer Graphics, vol. 374 of Studies in Computational Intelligence, pp. 139-155, Springer, Berlin, Germany, 2012.

[25] W. Chan and C. Armenakis, "3D building evacuation route modelling and visualization," International Archives of the Photogrammetry Remote Sensing and Spatial Information, vol. 2, no. 2, pp. 221-226.
[26] A. Ortony, G. L. Clore, and A. Collins, "The cognitive structure of emotions," Contemporary Sociology, vol. 18, no. 6, pp. 21472153, 1988.

[27] B. Liu, S. Chen, S.-Q. Xin, Y. He, Z. Liu, and J. Zhao, "An optimization-driven approach for computing geodesic paths on triangle meshes," Computer-Aided Design, vol. 90, pp. 105-112, 2017.

[28] A. Gingold R and J. Monaghan J, "Smoothed particle hydrodynamics: theory and application to non-spherical stars," Monthly Notices of the Royal Astronomical Society, vol. 181, no. 3, pp. 375389, 1977.

[29] L. B. Lucy, "A numerical approach to the testing of the fission hypothesis," The Astronomical Journal, vol. 82, pp. 1013-1024, 1977.

[30] H. D. Young, R. A. Freedman, A. L. Ford, and F. W. Sears, "Sears and zemanskys university physics with modern physics," June 2012.

[31] D. M. Mount, "ANN: A library for approximate nearest neighbor searching," pp. 783-792, 1998. 


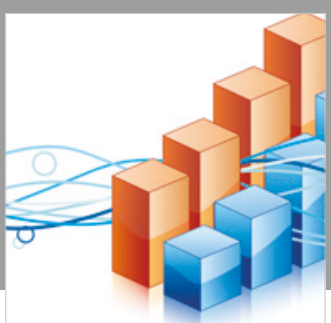

Advances in

Operations Research

\section{-n-m}
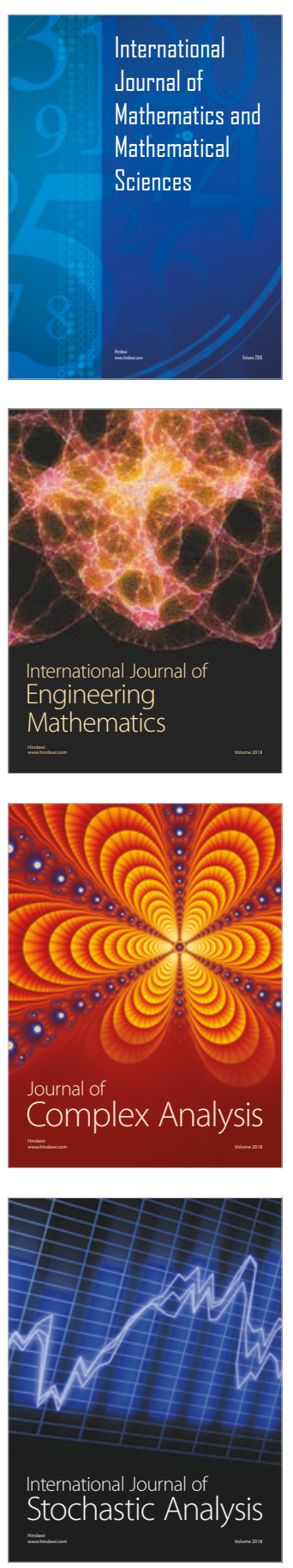
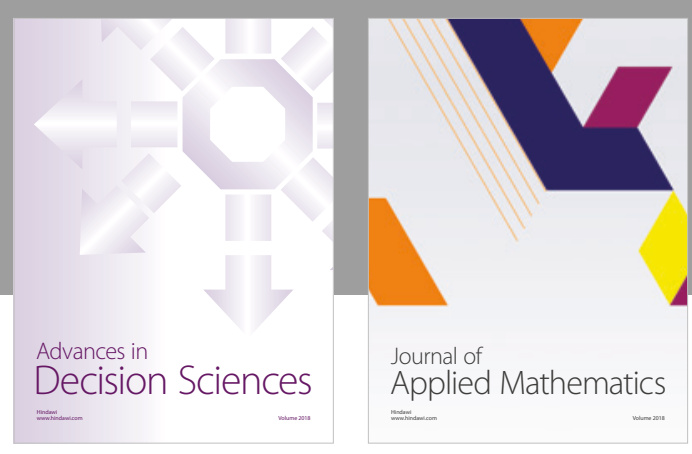

Journal of

Applied Mathematics
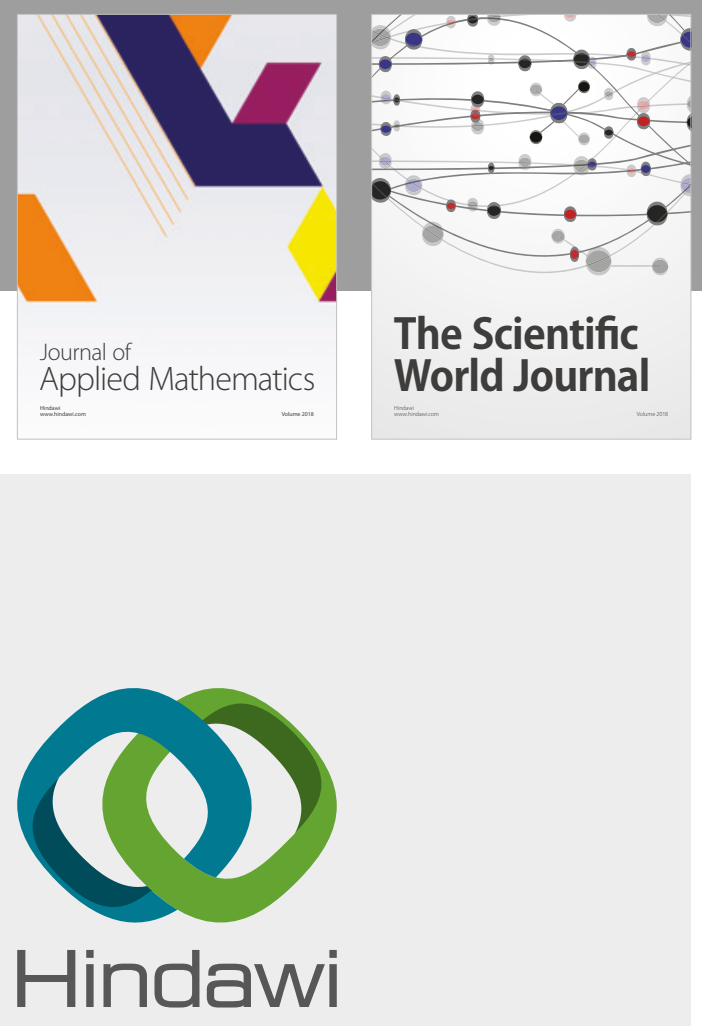

Submit your manuscripts at

www.hindawi.com

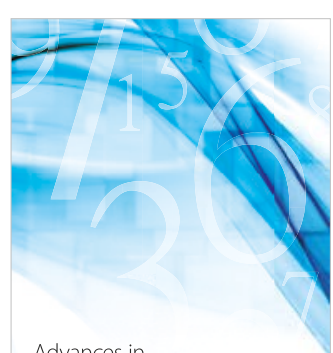

Advances in
Numerical Analysis
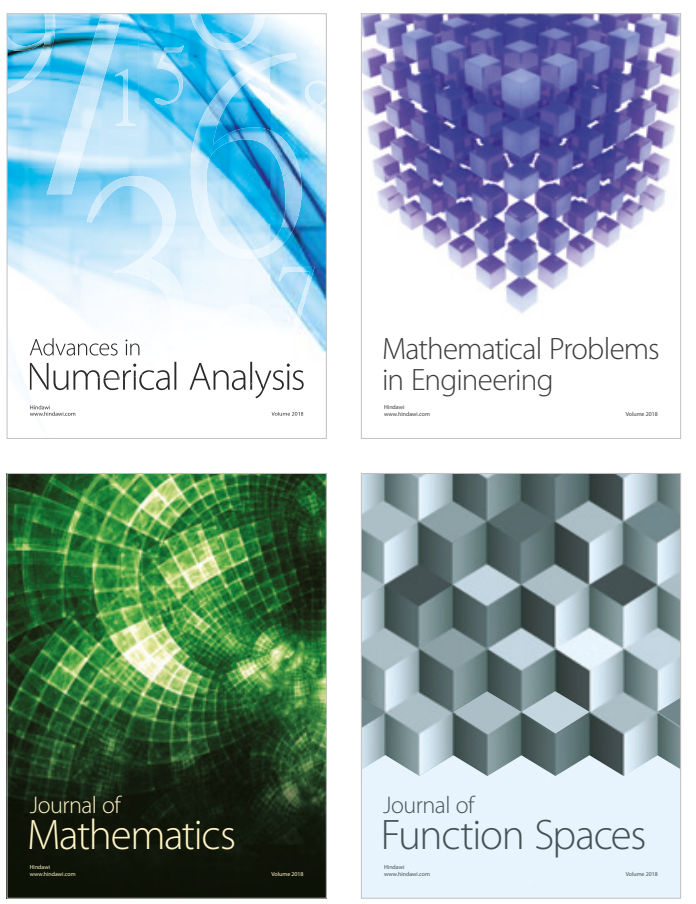

Mathematical Problems in Engineering

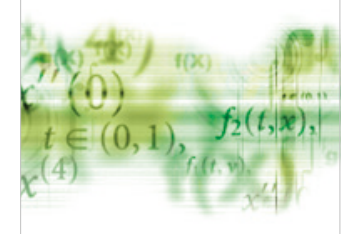

International Journal of

Differential Equations

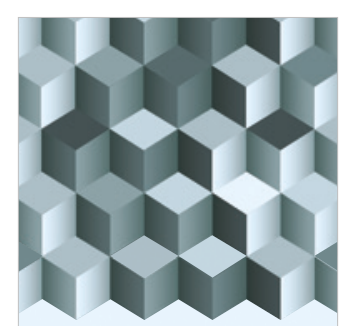

Journal of

Function Spaces

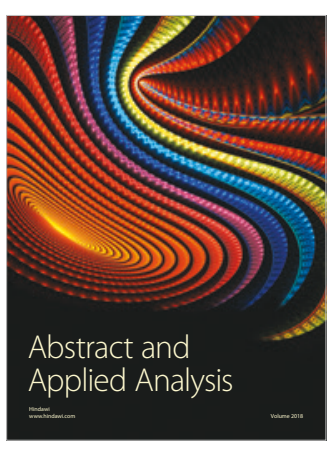

The Scientific

World Journal

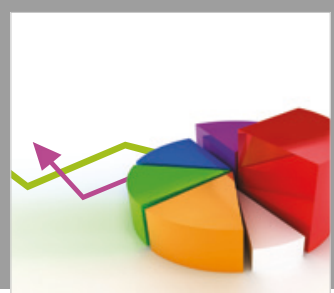

Journal of

Probability and Statistics
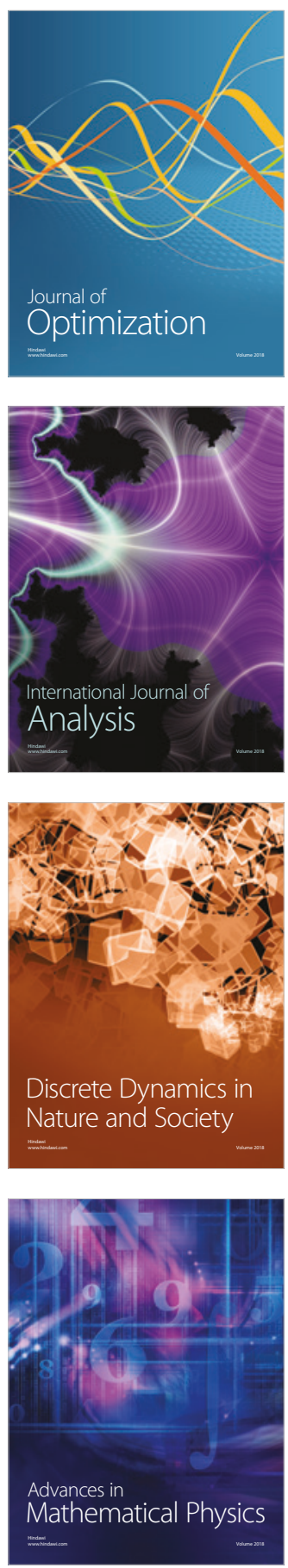